\title{
CORRECTION
}

\section{Author Correction: Tissue clearing of both hard and soft tissue organs with the PEGASOS method}

Dian Jing ${ }^{1,2}$, Shiwen Zhang ${ }^{1,2}$, Wenjing Luo ${ }^{1}$, Xiaofei Gao ${ }^{3}$, Yi Men ${ }^{1}$, Chi Ma ${ }^{1}$, Xiaohua Liu ${ }^{1}$, Yating Yi ${ }^{1,2}$, Abhijit Bugde $^{4}$, Bo O. Zhou ${ }^{5}$, Zhihe Zhao ${ }^{2}$, Quan Yuan ${ }^{2}$, Jian Q. Feng ${ }^{1}$, Liang Gao ${ }^{6}$, Woo-Ping Ge ${ }^{3}$ and Hu Zhao ${ }^{1}$

Cell Research (2019) 29:506; https://doi.org/10.1038/s41422-019-0180-5

Correction to: Cell Research https://doi.org/10.1038/s41422-0180049-z, published online 29 May 2018

In the initial published version of this article, there was an error in the "MATERIALS AND METHODS" section. The catalog number of PEGMMA500 for preparing tB-PEG dehydration solution and
BB-PEG clearing medium was listed as Sigma-Aldrich 409529. The correct catalog number should be Sigma-Aldrich 447943. The catalogue number for the same chemical provided in the Supplementary data S1 is correct. This correction does not affect the description of the results or the conclusions of this work.

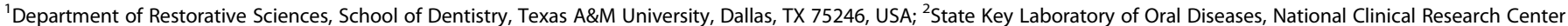

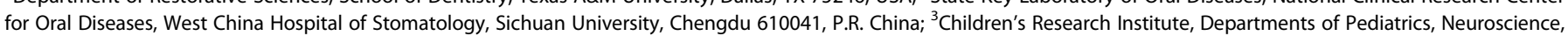

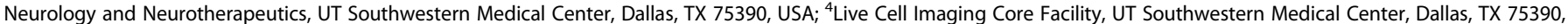

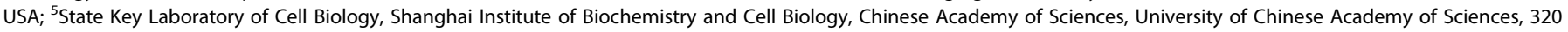
Yueyang Road, Shanghai 200031, P. R. China and ${ }^{6}$ Intelligent Imaging Innovations (3i) Inc., 3509 Ringsby Court, Denver, CO 80216, USA

Correspondence: Hu Zhao (hzhao@tamhsc.edu)

These authors contributed equally: Dian Jing, Shiwen Zhang, Wenjing Luo.

Published online: 20 May 2019 